\title{
A course on Moufang sets
}

\author{
Tom De Medts* $\quad$ Yoav Segev ${ }^{\dagger}$
}

\begin{abstract}
A Moufang set is essentially a doubly transitive permutation group such that the point stabilizer contains a normal subgroup which is regular on the remaining points. These regular normal subgroups are called the root groups and they are assumed to be conjugate and to generate the whole group.

Moufang sets play an significant role in the theory of buildings, they provide a tool to study linear algebraic groups of relative rank one, and they have (surprising) connections with other algebraic structures.

In these course notes we try to present the current approach to Moufang sets. We include examples, connections with related areas of mathematics and some proofs where we think it is instructive and within the scope of these notes.
\end{abstract}

Keywords: Moufang sets, BN-pairs, rank one groups, algebraic groups, Jordan algebras MSC 2000: 20E42, 51E24, 17C30, 20G15, 20B22

\section{Contents}

Introduction

1 Definition of a Moufang set $\quad 3$

1.1 Notation ....................... 3

1.2 Definition of a Moufang set . . . . . . . . . . . . 4

\footnotetext{
${ }^{*}$ Postdoctoral Fellow of the Research Foundation - Flanders (Belgium) (F.W.O.-Vlaanderen).

†Partially supported by the research group "Incidence Geometry" of Ghent University and by BSF grant no. 2004-083.
} 


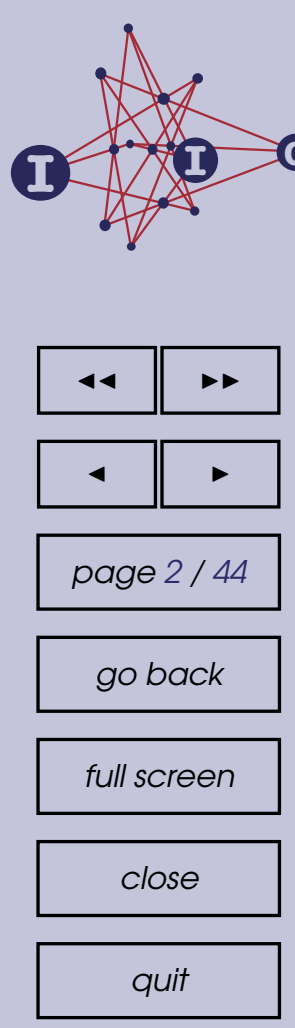

2 Motivation and situation $\quad 4$

2.1 Connection with BN-pairs of rank one . . . . . . . . . 4

2.2 Connection with abstract rank one groups . . . . . . . . . 6

2.3 Connection with higher rank groups . . . . . . . . . 8

2.4 Connection with linear algebraic groups of relative rank one . . . 9

2.5 Finite Moufang sets . . . . . . . . . . . . . 10

$\begin{array}{lll}3 & \text { Main construction } & 10\end{array}$

4 First properties of Moufang sets $\quad 13$

4.1 The $\mu$-maps . . . . . . . . . . . . . . . . 13

4.2 The Hua subgroup . . . . . . . . . . . . . . . . 14

4.3 Properties of the $\mu$-maps . . . . . . . . . . . . . 15

4.4 Connection between the $\mu$-maps and the Hua maps . . . . . . . 16

5 Examples of Moufang sets 17

$5.1 \mathbb{M}(k), k$ a commutative field . . . . . . . . . . . . . 17

$5.2 \mathbb{M}(D), D$ a skew field or an octonion division algebra . . . . . . . 19

$5.3 \mathbb{M}(J), J$ a quadratic Jordan division algebra . . . . . . . . . . . 19

5.4 Examples of Moufang sets with non-abelian root groups . . . . . 22

5.5 Connection with algebraic groups . . . . . . . . . . . 23

6 More advanced properties of Moufang sets 24

6.1 Identities in Moufang sets . . . . . . . . . . . . . 25

6.2 Root subgroups and the fixpoints of the Hua maps . . . . . . . . 26

7 Special Moufang sets $\quad 28$

7.1 Definition of special Moufang sets . . . . . . . . . . . . 28

7.2 The structure of the root groups . . . . . . . . . . . . . 29

7.3 The $\mu$-maps in special Moufang sets . . . . . . . . . . . . 32

7.4 The action of the Hua subgroup on the root groups . . . . . . . 34

7.5 The "special implies abelian" conjecture . . . . . . . . . . . . 36

7.6 The "special abelian implies Jordan algebra" conjecture . . . . . . 38

7.7 Finite special Moufang sets . . . . . . . . . . . . . 41 


\section{Introduction}

A Moufang set is essentially a doubly transitive permutation group $G$ such that the point stabilizer contains a normal subgroup which is regular on the remaining points. These regular normal subgroups are called the root groups and they are assumed to be conjugate and to generate $G$. (The root groups are not assumed to be nilpotent.)

J. Tits introduced this notion in the context of twin buildings, but it is in fact a tool to study absolutely simple algebraic groups of relative rank one; the Moufang sets are precisely the Moufang buildings of rank one. It turns out that this notion is related to other algebraic structures as well.

In these notes, we try to give the reader a sense of the "modern" approach to Moufang sets. We include examples and connections to related areas of mathematics; we provide detailed proofs where we think they could offer more insight into the theory, but for the same reason, we have omitted many details that can be found elsewhere and which are beyond the scope of this manuscript.

These notes have been used for a mini-course given by both authors, on the conference "Buildings and Groups" which took place in Ghent (Belgium), May 20-26, 2007. Our references for the material in this mini-course are [DS], [DS2], [DST], [DW], [S] and [SW] (we give more precise references at the beginning of the relevant sections).

We thank Pierre-Emmanuel Caprace, Shripad Garge, Max Horn, Guy Rousseau and Richard Weiss for valuable comments on an earlier version of this manuscript.

\section{Definition of a Moufang set}

\subsection{Notation}

We start by fixing some standard notation.

Notation 1.1.1. Let $G$ be a group and $p$ a prime.

(1) For $x, y \in G, x^{y}:=y^{-1} x y$ and $[x, y]:=x^{-1} y^{-1} x y$.

(2) When we write an inequality sign $H \leq G$, we always mean that $H$ is a subgroup of $G$ (while $S \subseteq G$ means that $S$ is a subset of $G$ ).

(3) For $S \subseteq G,\langle S\rangle$ is the subgroup generated by $S$.

(4) For a set $S$ we let $|S|$ be the cardinality of $S$.

(5) For an element $g \in G,|g|$ denotes the order of $G$.

(6) $G^{*}$ denotes the set of nontrivial elements of $G$. 



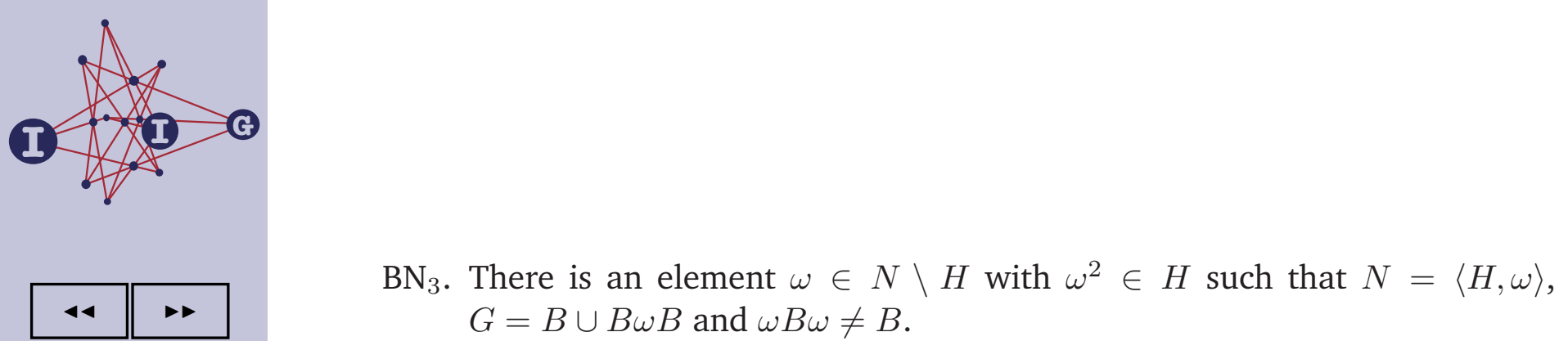

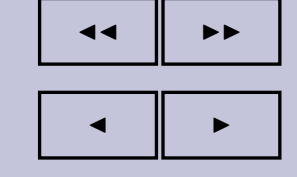

page $5 / 44$

go back

full screen

close

quit

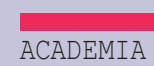

PRESS

WW

$\widehat{\underline{\text { IIIIIII }}}$

UNIVERSITEIT

GENT

The BN-pair is called split, if in addition the following axiom holds.

$\mathrm{BN}_{4}$. There exists a normal subgroup ${ }^{1} U \unlhd B$ such that $B=U \rtimes H$.

Any BN-pair (possibly non-split) is called saturated, if it also satisfies the following axiom. Note that we always have $H \leq B \cap B^{\omega}$.

$\mathrm{BN}_{5} . H=B \cap B^{\omega}$.

Remark 2.1.2. If a BN-pair $(B, N)$ for $G$ is not saturated, then $\left(B, N\left(B \cap B^{\omega}\right)\right)$ is a saturated BN-pair. However, if $(B, N)$ is split but not saturated, then its saturation $\left(B, N\left(B \cap B^{\omega}\right)\right)$ might be non-split.

Proposition 2.1.3. Let $G$ be a group with a saturated split BN-pair of rank one, and let $B, N, H, U$ and $\omega$ be as in Definition 2.1.1. Let

$$
X:=\left\{U^{g} \mid g \in G\right\}
$$

be the set of conjugates of $U$ in $G$. For any $x \in X$, denote the corresponding subgroup of $G$ (which is just $x$ itself!) by $V_{x}$. Then $\left(X,\left(V_{x}\right)_{x \in X}\right)$ is a Moufang set.

Proof. It is clear that each $V_{x}$ acts on $X$ by conjugation. We have to check whether the defining conditions $\mathbb{M}_{1}$ and $\mathbb{M}_{2}$ are satisfied. Condition $\mathbb{M}_{2}$ is clear, since each conjugate of $U$ is mapped, by conjugation, to some conjugate of $U$.

We claim that $N_{G}(U)=B$. Indeed, by $\mathrm{BN}_{4}, B$ normalizes $U$. So suppose that $U^{g}=U$ for some $g \in G \backslash B$. Then by $\mathrm{BN}_{3}, g=a \omega b$ for some $a, b \in B$, and hence $U^{a \omega b}=U$, implying $U^{\omega}=U$. But since $\omega$ normalizes $H$, this would imply $B^{\omega}=B$, contradicting the last statement of $\mathrm{BN}_{3}$.

We now proceed to show property $\mathbb{M}_{1}$. Let $x \in X$ be arbitrary, and write $V_{x}=x=U^{g}$. It is obvious that each $V_{x}$ fixes $x$ (i.e. itself) by conjugation. Assume that some element $v \in V_{x}$ fixes some $y \in X$, say $y=U^{h}$. Write $v=g^{-1} u g$ with $u \in U$; then $c:=h g^{-1} u g h^{-1}$ normalizes $U$, i.e. $c \in B$, and hence $c \in B \cap U^{g h^{-1}}$. Suppose that $y \neq x$, i.e. $g h^{-1} \notin B$, and use $\mathrm{BN}_{3}$ to write $g h^{-1}=a \omega b$ with $a, b \in B$. Then $B \cap U^{g h^{-1}} \neq 1$ implies $B^{\omega} \cap U \neq 1$. But by $\mathrm{BN}_{5}$, this would yield $U \cap H \neq 1$, contradicting $\mathrm{BN}_{4}$. We conclude that $V_{x}$ acts semi-regularly on $X \backslash\{x\}$.

Observe that $U^{\omega} \neq U$ implies $U \neq 1$; let $u \in U^{*}$. Then the three groups $U$, $U^{\omega}$ and $U^{\omega u}$ are pairwise distinct; hence $|X| \geq 3$.

\footnotetext{
${ }^{1}$ Some authors also require this subgroup to be nilpotent as part of the definition.
} 
We now claim that

$$
G=B \cup B \omega U
$$

Indeed, we have $H^{\omega}=H$ and hence $H=H^{\omega} \leq B^{\omega}$. It follows that $\omega H \subseteq B \omega$ and therefore $B \omega H U \subseteq B \omega U$. Since $H U=B$, we get $B \omega B=B \omega U$, and the claim follows.

It remains to show that $V_{x}$ is transitive on $X \backslash\{x\}$. Since $G$ is transitive by conjugation on $X$ it suffices to show that $U$ is transitive via conjugation on $X \backslash\{U\}$. It thus suffices to show that $X \backslash\{U\}=\left\{U^{\omega u} \mid u \in U\right\}$, but this is immediate from (2.1).

Remark 2.1.4. The group $G$ might be a non-trivial extension of the little projective group of the Moufang set. For example, if $G=\mathrm{SL}_{2}(k)$, then the little projective group of the corresponding Moufang set will be $\mathrm{PSL}_{2}(k)$. The crucial observation here is that, even though the groups $U^{g}$ act faithfully on $X$ and $G=\left\langle U^{g} \mid g \in G\right\rangle$, it might be that $G$ does not act faithfully. In other words, if $\psi_{x}$ is the natural injection from $V_{x}$ into $\operatorname{Sym}(X)$ for each $x \in X$, then the induced map $\psi$ from $G$ to $\operatorname{Sym}(X)$ might not be injective.

We now consider the converse.

Proposition 2.1.5. Let $\mathbb{M}=\left(X,\left(U_{x}\right)_{x \in X}\right)$ be an arbitrary Moufang set, with little projective group $G$. Let $0, \infty$ be two arbitrary elements of $X$, let $B:=G_{\infty}$, $N:=G_{\{0, \infty\}}, H:=G_{0, \infty}$ and $U:=U_{\infty}$ (i.e. the root group corresponding to $\infty$ ), and let $\omega$ an element of $G$ interchanging 0 and $\infty$. (Such elements $\omega$ always exist because $G$ is doubly transitive.) Then $B, N, \omega$ and $U$ satisfy all the axioms of a saturated split BN-pair of rank one.

Proof. It is straightforward to check conditions $\mathrm{BN}_{1}, \mathrm{BN}_{2}, \mathrm{BN}_{4}$ and $\mathrm{BN}_{5}$. To prove $\mathrm{BN}_{3}$, let $g \in G \backslash B$ be arbitrary, and let $x:=\infty g \neq \infty$. Let $u$ be the unique element of $U_{\infty}$ mapping 0 to $x$, and let $b:=g u^{-1} \omega^{-1}$. Then $\infty b=\infty$, hence $b \in B$ and $g=b \omega u \in B \omega B$. Moreover, $\omega B \omega=B^{\omega}=G_{0} \neq B$.

\subsection{Connection with abstract rank one groups}

The notion of abstract rank one groups was introduced by Franz Timmesfeld [Tim].

Definition 2.2.1. A group $G$ is called an abstract rank one group with unipotent subgroups $U$ and $V$, if $G=\langle U, V\rangle, U$ and $V$ are two distinct nilpotent subgroups of $G$, and 


$\mathrm{BN}_{2} . H:=B \cap N \unlhd N ;$

$\mathrm{BN}_{3}$. The group $W:=N / H$ has a generating set $R$ of involutions such that the following two relations hold for any $r \in R$ and any $w \in W$ :

- $r B w B \subseteq B w B \cup B r w B$;

- $r B r \neq B$.

The group $W$ is called the Weyl group of the BN-pair.

The BN-pair is called split, if there exists a normal subgroup ${ }^{2} U \unlhd B$ such that $B=U \rtimes H$. It is called saturated if $H=\bigcap_{w \in W} B^{w}$.

Any group with a BN-pair has a natural associated building on which it acts strongly transitively (i.e. transitive on the pairs consisting of an apartment and a chamber contained in it). In fact, a saturated BN-pair for a group $G$ is equivalent to a building on which $G$ acts strongly transitively. See, for example, [T74, Theorem 3.2.6 and Proposition 3.11].

\subsection{Connection with linear algebraic groups of relative rank one}

This subsection is not self-contained and requires knowledge about linear algebraic groups. The sole purpose of this subsection is to mention, very briefly and without proofs, one of the most important motivations for the study of Moufang sets.

Let $\mathbf{G}$ be an absolutely simple algebraic group defined over a field $k$, of $k$-rank one. Let $X$ denote the set of all $k$-parabolic subgroups of $\mathbf{G}$. Note that, since $\mathbf{G}$ has $k$-rank one, all elements of $X$ are conjugate under $\mathbf{G}(k)$. For each element $x \in X$, we let $U_{x}$ be the root subgroup of the $k$-parabolic subgroup $x$ (which coincides with the $k$-unipotent radical of $x)$. Let $\mathbf{G}^{+}(k)$ be the group generated by all these root subgroups. Then $\left(X,\left(U_{x}\right)_{x \in X}\right)$ is a Moufang set, on which $\mathbf{G}^{+}(k)$ modulo its center acts faithfully; we will denote it by $\mathbb{M}(\mathbf{G}, k)$.

The pairs of elements of $X$ are in one-to-one correspondence with the maximal $k$-split tori of $\mathbf{G}$. More precisely, for each $k$-split torus there are precisely two $k$-parabolic subgroups containing it, and every two $k$-parabolics contain a common $k$-split torus. If $S$ is such a maximal $k$-split torus, then $N(S) / Z(S)$ (which is the relative Weyl group of $\mathbf{G}$ over $k$ ) is a group of order 2.

Many of the exceptional algebraic groups of relative rank one are still poorly understood. It is our hope that the study of Moufang sets will eventually provide more insight into these groups.

\footnotetext{
${ }^{2}$ Some authors also require this subgroup to be nilpotent as part of the definition.
} 


\subsection{Finite Moufang sets}

Finite Moufang sets have already been studied and classified a long time before the notion of a Moufang set existed, as part of the classification of the finite simple groups, and more precisely in the study of finite split BN-pairs of rank one. Their classification was carried out by Suzuki [Su], Shult [Sh] and Peterfalvi [P] when the degree is odd, and by Hering, Kantor and Seitz [HKSe] when the degree is even. Some of these papers are hard and rely, in addition to the Feit-Thompson odd order theorem, on many other deep results in finite group theory.

It turns out that any finite Moufang set is either sharply two-transitive, or it is $\operatorname{PSL}_{2}(q), \operatorname{PSU}_{3}(q), \mathrm{Sz}(q) \cong{ }^{2} \mathrm{~B}_{2}(q)$ or $\operatorname{Re}(q) \cong{ }^{2} \mathrm{G}_{2}(q)$ for appropriate prime powers $q$.

In subsection 7.7, we will outline an elementary proof for the classification when the Moufang set is special, in which case the only possibility is $\operatorname{PSL}_{2}(q)$.

\section{Main construction}

Here we will describe how to construct an arbitrary Moufang set starting with a group $U$ and one permutation of the set $U^{*}$. The material of this section is taken from [DW].

Let $U$ be a group with composition + and identity 0 . (The operation + is not necessarily commutative. It will become clear in the examples why we have nevertheless chosen an additive notation.) Let $X$ denote the disjoint union of $U$ with $\{\infty\}$, where $\infty$ is a new symbol. For each $a \in U$, we denote

$$
\operatorname{Sym}(X) \ni \alpha_{a}:\left\{\begin{array}{l}
\infty \mapsto \infty \\
x \mapsto x+a
\end{array} \text { for all } a \in U .\right.
$$

Thus the map $a \mapsto \alpha_{a}$ is essentially the right regular representation of the group $U$. Let

$$
U_{\infty}:=\left\{\alpha_{a} \mid a \in U\right\}
$$

Now let $\tau$ be a permutation of $U^{*}$. We extend $\tau$ to an element of $\operatorname{Sym}(X)$ (which we also denote by $\tau$ ) by setting $0^{\tau}=\infty$ and $\infty^{\tau}=0$. Next we set

$$
U_{0}:=U_{\infty}^{\tau} \text { and } U_{a}:=U_{0}^{\alpha_{a}}
$$

for all $a \in U$. Let

$$
\mathbb{M}(U, \tau):=\left(X,\left(U_{x}\right)_{x \in X}\right)
$$






\section{First properties of Moufang sets}

From now on we assume that $\mathbb{M}=\mathbb{M}(U, \tau)$ is a Moufang set; in particular, by Theorem 3.5(i), the Hua maps $h_{a}$ act on $U$ as automorphisms. The material of subsections 4.1 and 4.2 can be found in [DW] and of subsections 4.3 and 4.4 in [DS].

go back

full screen

close

quit

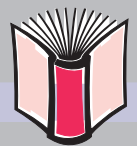

$\widehat{\underline{\text { IIIIII }}}$

\subsection{The $\mu$-maps}

We start by introducing certain permutations of $X$ which interchange the elements 0 and $\infty$. These maps play a central role in the analysis of Moufang sets.

Proposition 4.1.1. For each $a \in U^{*}$, there is a unique permutation $\mu_{a} \in U_{0}^{*} \alpha_{a} U_{0}^{*}$ interchanging 0 and $\infty$. This permutation will be denoted by $\mu_{a}$; we have

$$
\mu_{a}=\alpha_{(-a) \tau^{-1}}^{\tau} \cdot \alpha_{a} \cdot \alpha_{-\left(a \tau^{-1}\right)}^{\tau} .
$$

Proof. Let $\rho \in U_{0} \alpha_{a} U_{0}$ and assume that $0 \rho=\infty$ and $\infty \rho=0$. Write $\rho=\gamma_{x} \alpha_{a} \gamma_{y}$, with $\gamma_{x}, \gamma_{y} \in U_{0}$ as in Definition 3.3. Then $\infty=0 \rho=a \gamma_{y}=\left(a \tau^{-1}+y\right) \tau$. Hence $a \tau^{-1}+y=\infty \tau^{-1}=0$, so $y=-\left(a \tau^{-1}\right)$. Also,

$$
0=\infty \rho=\infty \gamma_{x} \alpha_{a} \gamma_{y}=\infty \tau^{-1} \alpha_{x} \tau \alpha_{a} \gamma_{y}=x \tau \alpha_{a} \gamma_{y}=(x \tau+a) \gamma_{y} .
$$

It follows that $x \tau+a=0 \gamma_{y}^{-1}=0$, so $x=(-a) \tau^{-1}$.

Since the $\mu$-maps of Proposition 4.1.1 are permutations interchanging 0 and $\infty$, any of them can take the role of the permutation $\tau$ :

Lemma 4.1.2. For each $a \in U^{*}$, we have $\mathbb{M}(U, \tau)=\mathbb{M}\left(U, \mu_{a}\right)$.

Proof. This follows from the fact that $\mu_{a} \in G, \mu_{a}$ interchanges 0 and $\infty$, and Remark 3.1.

Remark 4.1.3. It follows from Proposition 4.1.1 that, unlike the Hua-maps, the $\mu$-maps are independent of the choice of $\tau$.

Remark 4.1.4. If $\Delta$ is an arbitrary Moufang spherical building, then each group $X_{\alpha}=\left\langle U_{\alpha}, U_{-\alpha}\right\rangle$ generated by two opposite root groups is an abstract rank one group and therefore induces a Moufang set. The $\mu$-maps of this Moufang set are elements of $X_{\alpha}$, and hence act on all of $\Delta$. It turns out that these $\mu$-maps play an important role in the theory of Moufang buildings in general. For the rank two case (i.e. the case of Moufang polygons), see [TW, Chapter 6]. 


\subsection{The Hua subgroup}

We start with the definition of the Hua subgroup:

Definition 4.2.1. We define the Hua subgroup of $\mathbb{M}$ by

$$
H:=\left\langle\mu_{a} \mu_{b} \mid a, b \in U^{*}\right\rangle \text {. }
$$

Notice that $H \leq G_{0, \infty}$.

In light of Lemma 4.1.2, Theorem 3.5 and Proposition 4.3.1(2) below, we see that $\mu_{a} \mu_{b} \in \operatorname{Aut}(U)$, for all $a, b \in U^{*}$. Thus the Hua subgroup $H$ consists of automorphisms of $U$.

Lemma 4.2.2 ([DW, Theorem 3.1(ii)]). $H=G_{0, \infty}$.

Proof. By Lemma 4.1.2, we may and we will assume that $\tau=\mu_{e}$ for some $e \in U^{*}$. Let $K:=U_{0} H \leq G_{0}$, and let $Q:=K \tau \cup K U_{\infty}$. Note that $G=\left\langle U_{\infty}, \tau\right\rangle$; we want to show that $Q\left\langle U_{\infty}, \tau\right\rangle \subseteq Q$, which will imply that $Q=G$. Since only the trivial element in $\{\tau\} \cup U_{\infty}$ fixes 0 , this will imply that $G_{0}=K$, and then $G_{0, \infty}=K_{\infty}=H$.

So it remains to show that $Q U_{\infty} \subseteq Q$ and $Q \tau \subseteq Q$. We have $K \tau U_{\infty}=$ $K \tau U_{0}^{\tau}=K U_{0} \tau=K \tau$, which proves that $Q U_{\infty} \subseteq Q$. Clearly $(K \tau) \tau=K \tau^{2} \subseteq$ $K H=K$. Observe that $K H=K$ implies $K \tau=K \mu_{a}$ for all $a \in U^{*}$ (because $\mu_{a}^{-1}=\mu_{-a}$ for all $a \in U^{*}$, see Proposition 4.3.1(1) below), and hence by Proposition 4.1.1 and the fact that $K U_{0}=K$, we have $K \tau=K \mu_{a \tau}=K \alpha_{a \tau} \alpha_{-a}^{\tau}$ for all $a \in U^{*}$. It follows that

$$
K \alpha_{a} \tau=K \tau \cdot \alpha_{a}^{\tau}=K \alpha_{a \tau} \subseteq K U_{\infty}
$$

for all $a \in U^{*}$, which proves that $Q \tau \subseteq Q$.

Corollary 4.2.3. The following are equivalent:

(i) $G$ is sharply two-transitive;

(ii) $H=1$;

(iii) $\mu_{a}=\mu_{b}$ for all $a, b \in U^{*}$.

Proof. Since $G$ is always two-transitive, the equivalence between (i) and (ii) follows immediately from Lemma 4.2.2. The equivalence between (ii) and (iii) is immediate from the definition of $H$ and from Proposition 4.3.1(1) below.

Remark 4.2.4. Because of Corollary 4.2.3, the sharply two-transitive groups have a completely different behavior than the other Moufang sets. For that 




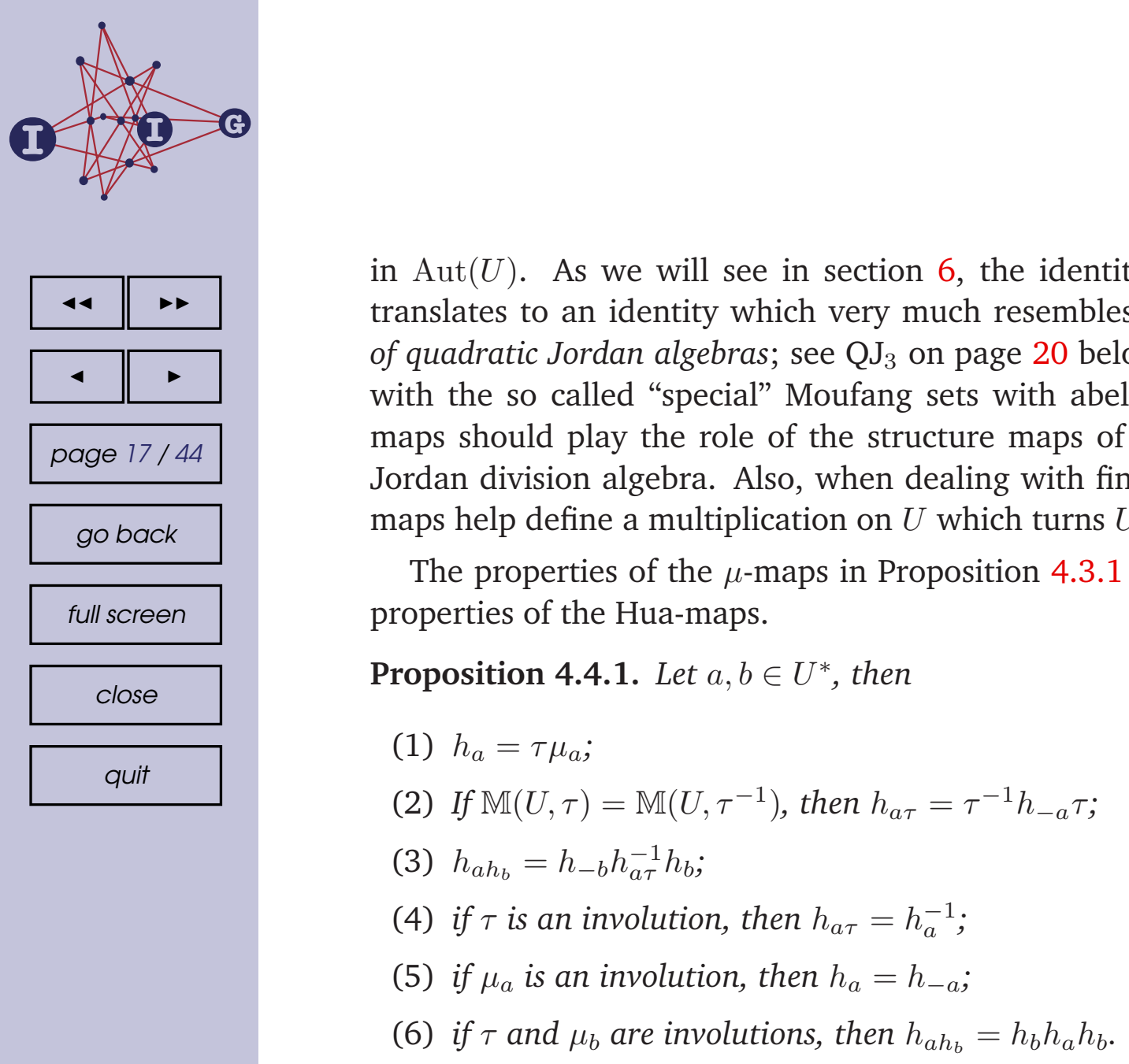

Proof. (1) This is Proposition 4.3.1(2).

(2) By (1) and Proposition 4.3.1(3),

$$
h_{a \tau}=\tau \mu_{a \tau}=\mu_{a}^{-1} \tau=\tau^{-1}\left(\tau \mu_{-a}\right) \tau=\tau^{-1} h_{-a} \tau .
$$

(3) Again by (1) and Proposition 4.3.1(3),

$$
h_{a h_{b}}=\tau \mu_{a \tau \mu_{b}}=\tau \mu_{b}^{-1} \mu_{a \tau}^{-1} \mu_{b}=\tau \mu_{-b} \mu_{a \tau}^{-1} \tau^{-1} \tau \mu_{b}=h_{-b} h_{a \tau}^{-1} h_{b} .
$$

(4) By (1) and by Proposition 4.3.1(3), $h_{a \tau}=\tau \mu_{a \tau}=\mu_{-a} \tau=h_{a}^{-1}$.

(5) By (1) and Proposition 4.3.1(1), $h_{a}=\tau \mu_{a}=\tau \mu_{-a}=h_{-a}$.

(6) This follows from (3) using (4) and (5). As we will see later, this identity is closely related to quadratic Jordan algebras; see subsection 5.3 below.

\section{Examples of Moufang sets}

\section{1. $\quad \mathbb{M}(k), k$ a commutative field}

We start by describing the easiest class of Moufang sets, namely those that arise from a commutative field $k$. The corresponding little projective group will turn 



\section{2. $\mathbb{M}(D), D$ a skew field or an octonion division algebra}

We will now generalize the previous example to so-called alternative division rings. These include all skew-fields, and in addition a family of non-associative division rings called octonion division algebras or Cayley-Dickson algebras.

Definition 5.2.1. A (not necessarily associative) ring $(D,+, \cdot)$ is called an alternative division ring if, for each $a \in D^{*}$, there exists some element $a^{-1} \in D^{*}$ such that $a \cdot a^{-1} b=b=b a^{-1} \cdot a$ for all $b \in D$.

full screen

close

quit

Even though this is a seemingly very weak associativity law, it turns out that almost all alternative division rings are skew fields:

Theorem 5.2.2. Let $D$ be an alternative division ring which is not a skew field. Then $D$ is 8-dimensional over its center $k$. It is a so-called octonion division algebra or Cayley-Dickson algebra. Its automorphism group is an anisotropic algebraic group of type $\mathrm{G}_{2}$ defined over $k$.

Proof. The fact that $D$ is a Cayley-Dickson algebra is due to Bruck and Kleinfeld [BrKl] when $\operatorname{char}(D) \neq 2$, and then completed by Kleinfeld [Kl] for the case where $\operatorname{char}(D)=2$. See, for example, [TW] for a characteristic-free proof. For the fact about the automorphism group in general characteristic, see [SV].

So let $D$ be an arbitrary alternative division ring. It can be shown that every subring generated by two elements is associative; in particular, the expression $a b a$ with $a, b \in D$ makes sense. Let $U:=(D,+)$, the additive group of $D$, and as in the previous example, let

$$
\tau: U^{*} \rightarrow U^{*}: x \mapsto-x^{-1} .
$$

Then the Hua maps are again given by

$$
x h_{a}=a-\left(a^{-1}-\left(a-x^{-1}\right)^{-1}\right)^{-1}
$$

for all $a \in U^{*}$ and all $x \in X$. The Hua identity now states that the right hand side of this expression is equal to $a x a$, and again it is clear that the restriction of each $h_{a}$ to $U$ is in $\operatorname{Aut}(U)$. Hence by Theorem 3.5, $\mathbb{M}(U, \tau)$ is a Moufang set. We will denote this Moufang set by $\mathbb{M}(D)$; it is sometimes called the projective Moufang set over $D$, and its little projective group is denoted by $\mathrm{PSL}_{2}(D)$.

\section{3. $\quad \mathbb{M}(J), J$ a quadratic Jordan division algebra}

All known examples of Moufang sets with abelian root groups (including the previous two examples) arise in the fashion which we will now describe. 
We first recall the definition of quadratic Jordan algebras, as introduced by K. McCrimmon [Mc1]. We will use the notation $W_{x}$ in place of the more common notation $U_{x}$, to avoid confusion with our notation for the root groups.

Let $k$ be an arbitrary commutative field, let $J$ be a vector space over $k$ of arbitrary dimension, and let $1 \in J^{*}$ be a distinguished element. For each $x \in J$, let $W_{x} \in \operatorname{End}_{k}(J)$, and assume that the map $W: J \rightarrow \operatorname{End}(J): x \mapsto W_{x}$ is quadratic, i.e.

$$
\begin{aligned}
& W_{x t}=W_{x} t^{2} \text { for all } t \in k \text {, and } \\
& \text { the map }(x, y) \mapsto W_{x, y} \text { is } k \text {-bilinear, }
\end{aligned}
$$

(note that we multiply scalars on the right) where

$$
W_{x, y}:=W_{x+y}-W_{x}-W_{y}
$$

for all $x, y \in J$. Let

$$
z V_{x, y}:=y W_{x, z}
$$

for all $x, y, z \in J$. Then the triple $(J, W, 1)$ is a quadratic Jordan algebra if the identities

$\mathrm{QJ}_{1} \cdot W_{1}=\mathrm{id}_{J}$

$\mathrm{QJ}_{2} \cdot W_{x} V_{x, y}=V_{y, x} W_{x}$;

$\mathrm{QJ}_{3} \cdot W_{y W_{x}}=W_{x} W_{y} W_{x} \quad$ ["the fundamental identity"]

hold strictly, i.e. if they continue to hold in all scalar extensions of $J$. (It suffices for them to hold in the polynomial extension $J_{k[t]}$ and this is automatically true if the base field $k$ has at least 4 elements.)

Any element $e \in J$ such that $W_{e}=\mathrm{id}_{J}$ is called an identity element. An element $x \in J$ is called invertible if there exists $y \in J$ such that

$$
y W_{x}=x \quad \text { and } \quad 1 W_{y} W_{x}=1 .
$$

In this case $y$ is called the inverse of $x$ and is denoted $y=x^{-1}$. By [Mc2, 6.1.2], an element $x \in J$ is invertible if and only if $W_{x}$ is invertible; we then have $W_{x}^{-1}=W_{x^{-1}}$. In particular,

$$
\left(x^{-1}\right)^{-1}=x \quad \text { and } \quad x^{-1}=x W_{x}^{-1} .
$$

If all elements in $J^{*}$ are invertible, then $(J, W, 1)$ is called a quadratic Jordan division algebra.

Now assume that $(J, W, 1)$ is an arbitrary quadratic Jordan division algebra. We will now construct a Moufang set $\mathbb{M}(J)$, in the same way as we did in the 



\subsection{Examples of Moufang sets with non-abelian root groups}

We will now briefly describe two different examples of Moufang sets with nonabelian root groups. There are many more interesting examples, but it is out of the scope of this course to go into more detail.

Example 5.4.1. Let $k$ be an arbitrary commutative field, and let $A$ be either a separable quadratic extension field of $k$, a quaternion division algebra over $k$, or an octonion division algebra over $k$. Let $\sigma$ be the standard involution of $A / k$, and let $N(a):=a a^{\sigma}$ and $T(a):=a+a^{\sigma}$ (for all $a \in A$ ) be the norm map and the trace map of $A / k$, respectively. Let

$$
U:=\{(a, b) \in A \times A \mid N(a)+T(b)=0\} .
$$

Then we can make $U$ into a (non-abelian) group by defining the group "addition"

$$
(a, b)+(c, d):=\left(a+c, b+d-c^{\sigma} a\right)
$$

for all $(a, b),(c, d) \in U$; it is easily checked that this is indeed a group, with neutral element $(0,0)$ and with the inverse given by $-(a, b)=\left(-a, b^{\sigma}\right)$. Now we define a permutation $\tau$ on $U^{*}$, by setting

$$
\tau(a, b)=\left(-a b^{-1}, b^{-1}\right)
$$

for all $(a, b) \in U^{*}$. Then $\mathbb{M}(U, \tau)$ is a Moufang set.

Remark 5.4.2. When $k=\mathrm{GF}(2)$ and $A=\mathrm{GF}(4)$, this gives the smallest example of a Moufang set with non-abelian root groups. It has $U \cong Q_{8}$, and hence $|X|=9$, and $G \cong \mathrm{PSU}_{3}(2)$.

Example 5.4.3. Let $k$ be an arbitrary commutative field with $\operatorname{char}(k)=3$ and admitting a Tits endomorphism $\theta$, i.e. an endomorphism such that $\left(x^{\theta}\right)^{\theta}=x^{3}$ for all $x \in k$. Let

$$
U:=\{(a, b, c) \mid a, b, c \in k\} .
$$

Then we can turn $U$ into a (non-abelian) group by defining the group "addition"

$$
\left(a, a^{\prime}, a^{\prime \prime}\right)+\left(b, b^{\prime}, b^{\prime \prime}\right)=\left(a+b, a^{\prime}+b^{\prime}+a b^{\theta}, a^{\prime \prime}+b^{\prime \prime}+a b^{\prime}-a^{\prime} b-a b^{1+\theta}\right)
$$

for all $a, a^{\prime}, a^{\prime \prime}, b, b^{\prime}, b^{\prime \prime} \in k$. We define a "norm" function on $U$ by setting

$$
N\left(a, a^{\prime}, a^{\prime \prime}\right):=-a^{4+2 \theta}-a a^{\prime \prime \theta}+a^{1+\theta} a^{\prime \theta}+a^{\prime \prime 2}+a^{\prime 1+\theta}-a^{\prime} a^{3+\theta}-a^{2} a^{\prime 2}
$$

for all $a, a^{\prime}, a^{\prime \prime} \in k$. We also set

$$
\begin{aligned}
& T_{1}\left(a, a^{\prime}, a^{\prime \prime}\right)=-a^{3+2 \theta}-{a^{\prime \prime}}^{\theta}+a^{\theta} a^{\prime}{ }^{\theta}+a^{\prime} a^{\prime \prime}+a a^{2}, \\
& T_{2}\left(a, a^{\prime}, a^{\prime \prime}\right)=-a^{3+\theta}+a^{\prime \theta}-a a^{\prime \prime}+a^{2} a^{\prime},
\end{aligned}
$$


for all $a, a^{\prime}, a^{\prime \prime} \in k$. Now let

$$
\tau: U^{*} \rightarrow U^{*}:\left(a, a^{\prime}, a^{\prime \prime}\right) \mapsto\left(\frac{-T_{1}\left(a, a^{\prime}, a^{\prime \prime}\right)}{N\left(a, a^{\prime}, a^{\prime \prime}\right)}, \frac{-T_{2}\left(a, a^{\prime}, a^{\prime \prime}\right)}{N\left(a, a^{\prime}, a^{\prime \prime}\right)}, \frac{-a^{\prime \prime}}{N\left(a, a^{\prime}, a^{\prime \prime}\right)}\right) .
$$

Then $\mathbb{M}(U, \tau)$ is a Moufang set. These are the so-called Ree-Tits Moufang sets. The corresponding little projective groups are the Ree groups of type ${ }^{2} \mathrm{G}_{2}$; in the finite case, these groups are sometimes considered to be the most complicated infinite class of finite simple groups.

full screen

close quit

Remark 5.4.4. A more natural but less direct way to describe these Moufang sets, is as the action of a certain subgroup of the centralizer of a polarity of a mixed Moufang hexagon $H\left(k, k^{\theta}\right)$ on the corresponding set of absolute points; see, for example, [DW2].

Remark 5.4.5. In example 5.4.1, the root groups have nilpotency class 2 , and in example 5.4.3, they have nilpotency class 3 . There is only one known other class of Moufang sets with root groups of nilpotency class 3, namely those arising from a polarity of a Moufang quadrangle of type $F_{4}$; see $[\mathrm{MV}]$. All other known examples of proper ${ }^{3}$ Moufang sets with non-abelian root groups have root groups of nilpotency class 2. On the other hand, it is not known whether there exist examples with non-nilpotent root groups, or with nilpotent root groups of higher nilpotency class.

\subsection{Connection with algebraic groups}

As we briefly explained in subsection 2.4, the theory of Moufang sets is motivated by its connection to linear algebraic groups of relative rank one. We point out (without going into detail) which of the previous examples arise from algebraic groups, and which do not.

The examples $\mathbb{M}(k)$, where $k$ is a commutative field, all arise from algebraic groups; in fact, they are the only examples arising from a split algebraic group of rank one defined over $k$, i.e. they arise from $\mathbf{G}=\mathrm{A}_{1}$.

The examples $\mathbb{M}(D)$, where $D$ is a skew field, arise from an algebraic group if and only if $D$ is finite-dimensional over its center $k$. In that case, they arise from a group with index

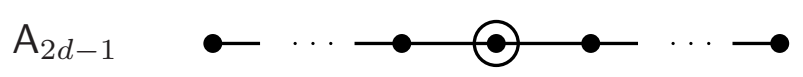

\footnotetext{
${ }^{3}$ See Remark 4.2.4.
} 





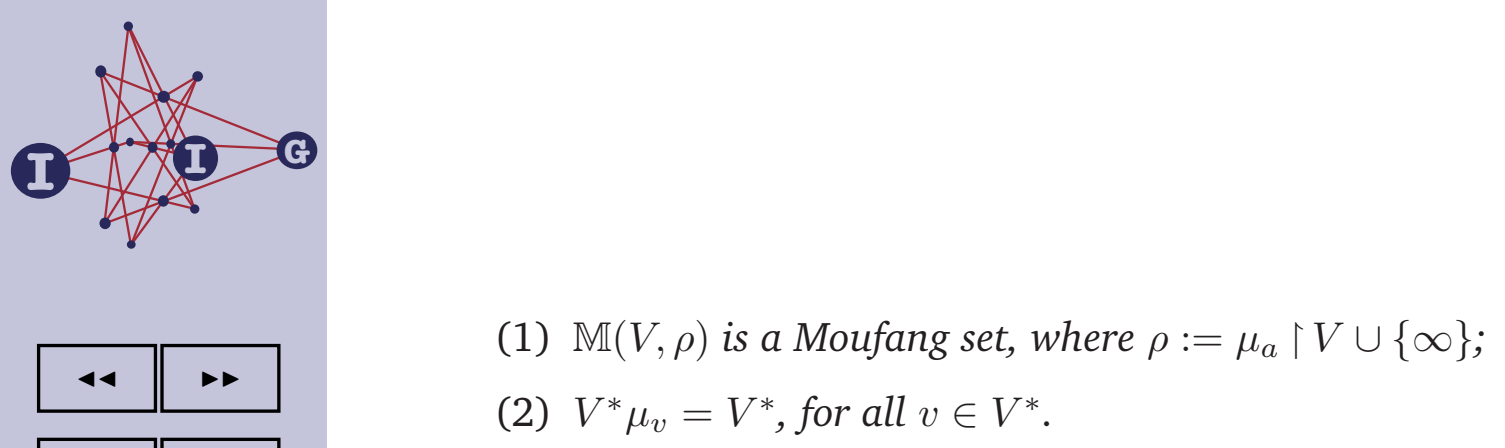

Proof. (1) Since $\mathbb{M}(U, \tau)=\mathbb{M}\left(U, \mu_{a}\right)$, we may assume that $\tau=\mu_{a}$. By the definition of the Hua-maps we now see that the Hua-maps of $\mathbb{M}(V, \rho)$ are the restriction of Hua-maps of $\mathbb{M}\left(U, \mu_{a}\right)$ to $V$, and hence they belong to $\operatorname{Aut}(V)$. By Theorem 3.5, $\mathbb{M}(V, \rho)$ is a Moufang set.

(2) This follows from the definition of the $\mu$-maps in Proposition 4.1.1.

One important place where root subgroups appear and where they turn out to be useful, is the following:

Lemma 6.2.3. For any $h \in H$, then $C_{U}(h)$ is a root subgroup.

Proof. Set $V:=C_{U}(h)$ and let $v, w \in V$. Then, by Proposition 4.3.1(4), we have $v \mu_{w} h=v h \mu_{w h}=v \mu_{w} \in V$, hence $V^{*} \mu_{w}=V^{*}$ and by definition $V$ is a root subgroup.

We will see that the notion of a root subgroups and Lemma 6.2.3 had already been used successfully for finite Moufang sets and for the "special if and only if abelian root groups" conjecture.

Notation 6.2.4. Let $0 \neq V \leq U$ be a root subgroup and let $x \in V^{*}$.

(1) We let $V_{\infty}:=\left\{\alpha_{v} \mid v \in V\right\}, V_{0}:=V_{\infty}^{\mu_{x}}$, and for $w \in V, V_{w}:=V_{0}^{\alpha_{w}}$. Notice that, since $\alpha_{v}^{\mu_{x} \mu_{y}}=\alpha_{v \mu_{x} \mu_{y}} \in V_{\infty}$ for all $v \in V$ and all $x, y \in V^{*}$, the definition of $V_{y}, y \in V \cup\{\infty\}$ is independent of the choice of $x$.

(2) We let $G(V):=\left\langle\alpha_{v}, \mu_{v} \mid v \in V^{*}\right\rangle, N(V):=\left\langle\mu_{v} \mid v \in V^{*}\right\rangle, H(V):=$ $\left\langle\mu_{v} \mu_{w} \mid v, w \in V^{*}\right\rangle$ and $X(V):=V \cup\{\infty\}$.

Definition 6.2.5. A group $G$ is called a generalized abstract rank one group with unipotent subgroups $A$ and $B$, if $G=\langle A, B\rangle, A$ and $B$ are two different subgroups of $G$, and for each $a \in A^{*}$, there exists an element $b \in B^{*}$ such that $A^{b}=B^{a}$, and conversely. Note that the only difference with Timmesfeld's definition of an abstract rank one group (as given in Definition 2.2.1) is that we do not require $A$ and $B$ to be nilpotent.

Lemma 6.2.6. Let $0 \neq V \leq U$ be root subgroup, set $\mathcal{X}:=X(V)$ and let $\mathcal{G}:=$ $G_{\{\mathcal{X}\}}$ and $\mathfrak{G}:=G(V)$. Then

(1) $\mathfrak{G}$ is a generalized rank one group with unipotent subgroups $V_{\infty}$ and $V_{0}$;

(2) $\mathfrak{G} \unlhd \mathcal{G}$ and $\mathcal{G}=\mathfrak{G} H_{\{V\}}$; 


It thus remains to show (7.1). The proof is by induction on $n$. For $n=1$, this is Proposition 7.3.1(2). Assume that $a \cdot(n+1) \neq 0$. Note that if $a \cdot n=0$, then $a \cdot(n+1) \mu_{a} \cdot(n+1)=a \mu_{a} \cdot(n+1)=-a$, so we may assume that $a \cdot n \neq 0$; hence by the induction hypothesis, equations (7.1), (7.2) and parts (1) and (2) hold for $n$. Notice that also $a \cdot(n+1) n \neq 0$, because otherwise we would get $(a \cdot n) \cdot n=(-a) \cdot n$, but then, by the uniqueness in part (1) (which holds for $n$ ), $a \cdot n=-a$, which is false. Hence $a \cdot(n+1) \cdot \frac{1}{n}$ makes sense.

By Proposition 4.3.1(8) and Lemma 7.1.4(v), $\mu_{-a}=\alpha_{a} \mu_{-a} \alpha_{a} \mu_{-a} \alpha_{a}$. Hence, using equation (7.2) (which holds for $n$ by induction) we get

$$
\begin{aligned}
-\left((a \cdot(n+1)) \mu_{-a}\right) & =((-a) \cdot(n+1)) \mu_{-a} \\
& =((-a) \cdot(n+1)) \alpha_{a} \mu_{-a} \alpha_{a} \mu_{-a} \alpha_{a} \\
& =((-a) \cdot n) \mu_{-a} \alpha_{a} \mu_{-a} \alpha_{a} \\
& \text { induction }\left(a \cdot \frac{1}{n}+a\right) \mu_{-a} \alpha_{a} \\
& =\left(a \cdot(n+1) \cdot \frac{1}{n}\right) \mu_{-a} \alpha_{a} \\
& \stackrel{\text { induction }}{=}\left(a \cdot(n+1) \mu_{-a}\right) \cdot n+a .
\end{aligned}
$$

Hence, $(a \cdot(n+1)) \mu_{-a} \cdot(n+1)=-a$. This completes the proof of (1) and (2).

Remark 7.2.3. The statement in Proposition 7.2.2(5) is equivalent to stating that $U$ is a vector space over some field $\mathbb{F}$, where $\mathbb{F}$ can be chosen to be either a finite field $\operatorname{GF}(p)$ or the field of rationals $\mathbb{Q}$. This field $\mathbb{F}$ is called the prime field of $U$, and such a group $U$ is sometimes called a vector group.

Remark 7.2.4. Notice that Proposition 7.2.2 says that the order of any element in $U^{*}$ is either a prime or $\infty$ and that $U$ has very interesting unique divisibility properties, i.e., if $a \in U^{*}$ is such that $a \cdot n \neq 0$, then $\left(a \rho^{-1} \cdot n\right) \rho$ is the unique $n$-th root of $a$ in $U$.

The next lemma shows that the structure of centralizers in $U$ is very restricted.

Lemma 7.2.5. (1) If $a \in U^{*}$ is an element whose order is a prime $p$, then $C_{U}(a)$ is a group of exponent $p$;

(2) if $a \in U^{*}$ is of infinite order, then $C_{U}(a)$ is a torsion-free uniquely divisible group.

Proof. (1) Let $b \in C_{U}(a)$ and assume that the order of $b$ is not $p$. Then the order of $a+b$ is not $p$ and by (1) we have

$$
\left((a+b) \cdot \frac{1}{p}-b \cdot \frac{1}{p}\right) \cdot p=a,
$$

contradicting the fact that $a$ has no $p$-root in $U$ (by Proposition 7.2.2(4)). 

It follows that $x \mu_{-a}=x \mu_{a}$ for all $x$, and hence $\mu_{a}^{-1}=\mu_{-a}=\mu_{a}$.

Remark 7.3.2. In Theorem 7.5.2 below, we will see that (12) is actually equivalent to the assertion that $U$ is abelian.

\subsection{The action of the Hua subgroup on the root groups}

The following structural theorem turns out to be very useful in proving various results about special Moufang sets.

Theorem 7.4.1 ([SW]). Let $W \leq U$ be a nontrivial $H$-invariant subgroup. Then either $U$ is an elementary abelian 2-group, or $W=U$.

Sketch of proof. Let $W$ be an $H$-invariant subgroup of $U$. First we notice that

$$
W^{*} \mu_{u}=W^{*} \text { for all } u \in U^{*} .
$$

This is because $w \mu_{u}=(-w) \mu_{w} \mu_{u}$, for all $w \in W^{*}$.

Step 1. Let $a \in W^{*}$ and $b \in U$. If $b+a+b \in W$, then $b \cdot n+a+b \cdot n \in W$ for all $n \in \mathbb{Z}$.

Step 2. As a corollary we get:

Let $a \in W^{*}$ and $b \in U^{*}$. If $a+b \neq 0$, then $b \cdot n+a \mu_{a+b}+b \cdot n \in W$ for all $n \in \mathbb{Z}$.

Proof of Step 2. As $a \in W^{*}$, equation (7.4) says that $a \mu_{b}$ and $a \mu_{a+b}$ are also in $W$, so $-a+a \mu_{b} \in W$. Then, by Proposition 7.3.1(5), $b+a \mu_{a+b}+b=$ $-a+a \mu_{b} \in W$, so Step 2 follows from Step 1 .

Step 3. Assume $W$ is normal in $U$. Then either $U$ is an elementary abelian 2-group or $W=U$.

Proof of Step 3. We repeatedly use the fact that for $x \in U^{*}, W^{*} \mu_{x}=W^{*}$ (and hence if $u \in U \backslash W$, then $u \mu_{x} \notin W$ ). We assume that $W \neq U$ and we show that $U$ is an elementary abelian 2-group.

First we show that

$$
\text { if } w \in W \text { and } w \cdot 2 \neq 0 \text {, then } w \cdot \frac{1}{2} \in W \text {. }
$$

Let $w \in W$ such that $w \cdot 2 \neq 0$. Then, by Proposition 7.2.2(2), with $\rho=\mu_{w}$, we have $w \cdot \frac{1}{2}=((-w) \cdot 2) \mu_{w}$. Hence $w \cdot \frac{1}{2} \in W$. Next we claim that

$$
\text { if } u \in U \text { and } u \cdot 2 \neq 0 \text {, then } u \in W \text {. }
$$



Consider the equality

$$
a \mu_{a+b}=-b+a+a \mu_{b}-b=a+b+a \mu_{b}-b .
$$

Since $a+b \in \operatorname{Inv}(U)$ (because $a$ inverts $b$ ), it follows from (*) that $a$ commutes with $a \mu_{a+b}$ so $a$ commutes with $b+a \mu_{b}-b$. Conjugating by $b$ we see that $a \mu_{b}$ commutes with $-b+a+b$, hence

$$
\text { if } a \text { inverts } x \in U^{*} \backslash \operatorname{Inv}(U) \text {, then } a \mu_{x} \text { commutes with }-x+a+x \text {. }
$$

In what follows we will use the following facts from Proposition 7.3.1(8):

$$
(b \cdot \gamma) \mu_{b \cdot \delta}=-b \cdot \frac{\delta^{2}}{\gamma}, \quad \mu_{b \cdot \gamma}^{\mu_{b \cdot \delta}}=\mu_{b \frac{\delta^{2}}{\gamma}}
$$

for all $\gamma, \delta \in \mathbb{Q}$ such that $b \cdot \gamma, b \cdot \delta$ are well defined. Notice that the uniqueness of roots in $U$ implies that $a$ inverts $b \cdot \gamma$, for every $\gamma \in \mathbb{Q}$ for which $b \cdot \gamma$ is well defined. Let now $\alpha, \beta \in \mathbb{Q}$ such that $b \cdot \alpha$ and $b \cdot \beta$ are well defined. From equation (7.5) we get

$$
a \mu_{b \cdot \alpha} \text { commutes with }-b \cdot \alpha+a+b \cdot \alpha \text {. }
$$

Applying $\mu_{-b \cdot \alpha} \mu_{b \cdot \beta} \in \operatorname{Aut}(U)$ to equation (7.7) we get

$$
a \mu_{b \cdot \beta} \text { commutes with }-b \cdot \frac{\beta^{2}}{\alpha}+a \mu_{-b \cdot \alpha} \mu_{b \cdot \beta}+b \cdot \frac{\beta^{2}}{\alpha} .
$$

Replacing in this last equality $\beta$ with $\alpha$ and $\alpha$ with $-\beta$ we get

$$
a \mu_{b \cdot \alpha} \text { commutes with } b \cdot \frac{\alpha^{2}}{\beta}+a \mu_{b \cdot \beta} \mu_{b \cdot \alpha}-b \cdot \frac{\alpha^{2}}{\beta} \text {. }
$$

From equations (7.7) and (7.8) using $(* *)$ we see that

$$
-b \cdot \alpha+a+b \cdot \alpha \text { commutes with } b \cdot \frac{\alpha^{2}}{\beta}+a \mu_{b \beta} \mu_{b \alpha}-b \cdot \frac{\alpha^{2}}{\beta}
$$

and after conjugating by $-b \alpha$ we get

$$
a \text { commutes with } a \mu_{b \cdot \beta} \mu_{b \cdot \alpha}-b \cdot\left(\alpha+\frac{\alpha^{2}}{\beta}\right) \cdot 2 .
$$

Notice that we have used (7.6) which implies that $a \mu_{b \cdot \beta} \mu_{b \cdot \alpha}$ inverts $b$ (because $\left.\mu_{b \cdot \beta} \mu_{b \cdot \alpha} \in \operatorname{Aut}(U)\right)$. Since $a$ and $a \mu_{b \beta} \mu_{b \alpha}$ invert $b, a+a \mu_{b \cdot \beta} \mu_{b \cdot \alpha}$ centralizes $b$. But by equation (7.9), $a$ commutes with $c:=a+a \mu_{b \cdot \beta} \mu_{b \cdot \alpha}-b \cdot\left(\alpha+\frac{\alpha^{2}}{\beta}\right) \cdot 2$ and $c$ commutes with $b$. Hence, if $c \neq 0$, then, by $(* *), c$ is an involution, and hence $b$ is an involution. We have thus shown that

$$
a \mu_{b \cdot \beta} \mu_{b \cdot \alpha}=a+b \cdot\left(\alpha+\frac{\alpha^{2}}{\beta}\right) \cdot 2 .
$$


Taking in equation (7.10) $\alpha=\beta=-1$ we get

$$
a \mu_{-b}^{2}=a-b \cdot 4 \text {. }
$$

But taking in equation (7.10) $\beta=-1$ and $\alpha=2$ we also get

$$
a \mu_{-b} \mu_{b \cdot 2}=a-b \cdot 4
$$

Hence $a \mu_{-b}^{2}=a \mu_{-b} \mu_{b \cdot 2}$. Applying $\mu_{b}$ on both sides of this equality and using equation (7.6) we obtain $a \mu_{-b}=a \mu_{b \cdot \frac{1}{2}}$ or

$$
a=a \mu_{b \cdot \frac{1}{2}} \mu_{b}
$$

But from equations (7.10) and (7.13) we get

$$
a=a \mu_{b \cdot \frac{1}{2}} \mu_{b}=a+b \cdot 6 .
$$

so $b \cdot 6=0$. Since the order of $b$ is a prime (see Proposition 7.2.2(4)) and $b \notin \operatorname{Inv}(U)$, we see that $b \cdot 3=0$. But then, by Proposition 7.3.1(11), $\mu_{b}^{2}=\mu_{-b}^{2}$. However, by equation (7.10), $a \mu_{b}^{2}=a+b \cdot 4$ whereas $a \mu_{-b}^{2}=a-b \cdot 4$, so $b \cdot 8=0$. This is a contradiction and the proof this step is complete.

Step 2. Let $b \in U^{*}$. We will show that $b \in \operatorname{Inv}(U)$. Assume not and let $a \in \operatorname{Inv}(U)$, then $a \mu_{a+b}=-b+a+a \mu_{b}-b$, and conjugating by $b$ we get that $-b \cdot 2+a+a \mu_{b} \in \operatorname{Inv}(U)$. Thus $a \mu_{b}$ inverts $-b \cdot 2+a$, so, by Step $1,-b \cdot 2+a$ is an involution. It follows that $a$ inverts $-b \cdot 2$ and hence $a$ inverts $b$. But then, by Step $1, b$ is an involution, a contradiction. This completes the proof of Theorem 7.5.1.

\subsection{The "special abelian implies Jordan algebra" conjecture}

In this section we assume that $U$ is abelian. Hence, by Proposition 7.3.1(13),

$$
\mu_{a}^{2}=1, \text { for all } a \in U^{*} .
$$

We assume that $\tau=\mu_{e}$ for some $e \in U^{*}$, but occasionally $e$ may vary. Recall that by Remark 7.2.3, $U$ is a vector space over a prime field $\mathbb{F}$. Recall also that by Theorem 3.5, $h_{a}$ is an invertible $\mathbb{F}$-linear transformation of $U$, for all $a \in U^{*}$.

The main conjecture here is:

Conjecture 7.6.1. There exists a field extension $\mathbb{K} / \mathbb{F}$ such that

(1) $U$ is a vector space over $\mathbb{K}$;

(2) $h_{a} \in \operatorname{End}_{\mathbb{K}}(U)$, for all $a \in U^{*}$; 


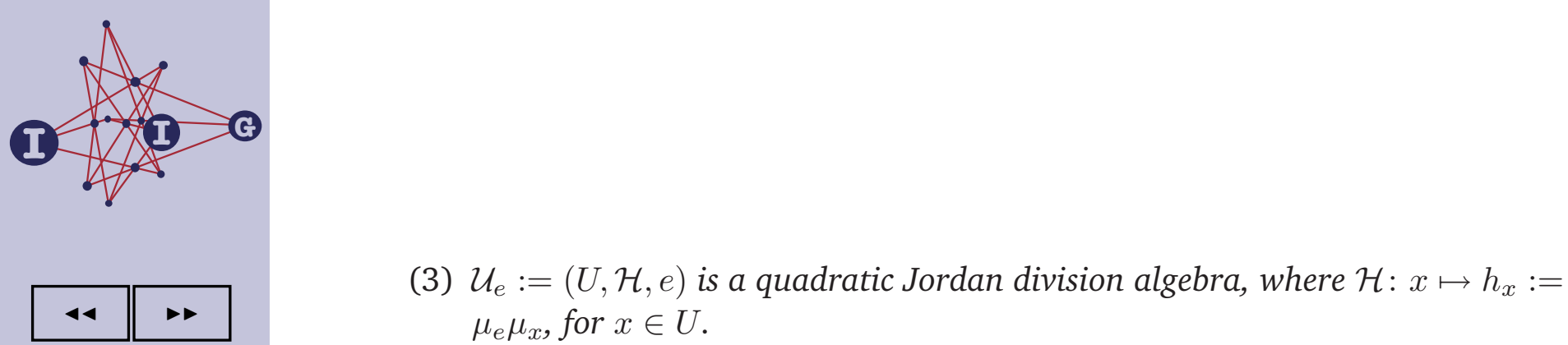

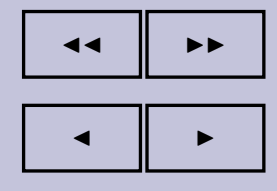

page 39 / 44

go back

full screen

close

quit

Notice that since quadratic Jordan division algebras have been classified (see Remark 5.3.2), this will yield a complete classification of special Moufang sets with abelian root groups.

One possible candidate for the field $\mathbb{K}$ of Conjecture 7.6.1 is

$$
\mathbb{K}:=Z\left(C_{\operatorname{End}_{\mathbb{F}}(U)}(H)\right), \quad \text { if } \operatorname{char}(\mathbb{F}) \neq 2 .
$$

Notice that since $H$ acts irreducibly on $U, \mathbb{K}$ is a commutative field. Note that any quadratic Jordan algebra over $\mathbb{K}$ is also a quadratic Jordan algebra over $\mathbb{F}$, so another possibility is just to take $\mathbb{K}=\mathbb{F}$.

Of course $\mathcal{U}_{e}$ depends on the choice of $e$, because the Hua-maps $h_{a}=\mu_{e} \mu_{a}$ depend on $e$. Inspired by the theory of Jordan algebras, it makes sense to call $\mathcal{U}_{e}$ an isotope.

We define

$$
h_{a, b}:=h_{a+b}-h_{a}-h_{b}
$$

for all $a, b \in U$, with the convention that $h_{0}$ is the zero map. Recall that $(U, \mathcal{H}, e)$ is a quadratic Jordan division algebra if and only if

(Quadratic) $\mathcal{H}$ is quadratic, i.e.

(i) $h_{x t}=h_{x} t^{2}$ for all $t \in \mathbb{K}$;

(ii) the map $(x, y) \mapsto h_{x, y}$ is $k$-bilinear.

(QJ axioms) The following identities hold strictly:

$$
\begin{aligned}
& \mathrm{QJ}_{1} \cdot h_{e}=\mathrm{id}_{U} ; \\
& \mathrm{QJ}_{2} \cdot a h_{c, b} h_{a}=c h_{a, b h_{a}}, \quad \text { for all } a, b, c \in U \text {; } \\
& \mathrm{QJ}_{3} . h_{b h_{a}}=h_{a} h_{b} h_{a}, \quad \text { for all } a, b \in U .
\end{aligned}
$$

Notice that axiom $\mathrm{QJ}_{2}$ actually says that $h_{a} V_{a, c}=V_{c, a} h_{a}$, where $b V_{a, c}=c h_{a, b}$, hence $b h_{a} V_{a, c}=c h_{a, b h_{a}}$ and $b V_{c, a} h_{a}=a h_{c, b} h_{a}$.

Of course, $\mathrm{QJ}_{1}$ obviously holds. Also, $\mathrm{QJ}_{3}$ is Proposition 4.4.1(6), and $h_{a \cdot s}=$ $h_{a} \cdot s^{2}$ for all $a \in U^{*}$ and $s \in \mathbb{F}$. So we can see that some of the axioms defining a quadratic Jordan division algebra already hold. What is actually missing is:

Missing axioms: The biadditivity of $(x, y) \mapsto h_{x, y}$ and $\mathrm{QJ}_{2}$.

Our first observation is the following. 



\subsection{Finite special Moufang sets}

The techniques that were introduced above are useful to give a short and direct proof for the (known) classification of finite special Moufang sets.

Notation 7.7.1. For any prime power $q$, we write $\mathbb{M}(q):=\mathbb{M}(\mathrm{GF}(q))$, where $\mathbb{M}(k)$ is the projective Moufang set over the commutative field $k$ as defined in subsection 5.1 .

The proof of the following Theorem uses the Feit-Thompson "Odd Order Theorem" and Glauberman's $Z^{*}$-Theorem. Otherwise, it is self contained. We will only sketch the proof very briefly, and we refer to [DS2] for more details.

Theorem 7.7.2. Let $\mathbb{M}(U, \tau)$ be a finite special Moufang set such that $|U|=q$ is even. Then $q$ is a power of $2, U$ is elementary abelian and $\mathbb{M}(U, \tau) \cong \mathbb{M}(q)$.

Sketch of proof.

Step 1. By Theorem 7.5.1, $U$ is elementary abelian.

Step 2. $|H|$ is odd and $H$ is transitive on $U^{*}$.

Sketch of proof of Step 2. Let

$$
\mathcal{I}:=\bigcup_{x \in X} U_{x}^{*}
$$

First we show that

$\mathcal{I}$ is a conjugacy class of involutions in $G$.

From (7.16) it follows that $H$ is transitive on $U^{*}$, and therefore, by Proposition 4.3.1(4) and Proposition 7.3.1(7),

$$
\left\{\mu_{a} \mid a \in U^{*}\right\} \text { is a conjugacy class of involutions in } N \text {. }
$$

Notice however that for $a, b \in U^{*}$ with $a \neq b,\left[\mu_{a}, \mu_{b}\right] \neq 1$, because $\mu_{a}^{\mu_{b}}=\mu_{a \mu_{b}}$, so if $\mu_{a \mu_{b}}=\mu_{a}, a \mu_{b}=a$, but $b$ is the unique fixed point of $\mu_{b}$, because $\mu_{b}$ is conjugate to $\alpha_{b}$.

By (7.16) and Glauberman's $Z^{*}$-Theorem, $\mu_{a} \mu_{b} \in O_{2^{\prime}}(N)$, for all $a, b \in U^{*}$, where $O_{2^{\prime}}(N)$ is the largest normal subgroup of odd order of $N$. However, $H=\left\langle\mu_{a} \mu_{b} \mid a, b \in U^{*}\right\rangle$, so $H \leq O_{2^{\prime}}(N)$ and hence $|H|$ is odd. 
Step 3. $H$ is cyclic.

Proof of Step 3. We use the following very useful Lemma of Peterfalvi:

Lemma (Peterfalvi [P2]). Let $p$ be an odd prime, and suppose that $P$ is a p-group acting faithfully on $U$ with $C_{U}(P)=0$. If $\left|C_{P}(a)\right|=\left|C_{P}(b)\right|$ for all $a, b \in U^{*}$, then $P$ is cyclic.

Now by Step 2, $H$ is solvable and since $H$ is transitive on $U^{*}$,

$$
\left|C_{O_{p}(H)}(e)\right|=\left|C_{O_{p}(H)}(f)\right|, \quad \text { for all primes } p \text { and all } e, f \in U^{*} .
$$

Hence by Peterfalvi's Lemma, $O_{p}(H)$ is cyclic for all primes $p$, so the Fitting subgroup $F(H)=\prod_{p \text { prime }} O_{p}(H)$ is cyclic and hence $\langle h\rangle \unlhd H$, for all $h \in$ $F(H)^{*}$. Since $H$ is transitive on $U^{*}$,

$$
C_{U}(h)=0 \text {, for all } h \in F(H)^{*} .
$$

This implies $C_{F(H)}\left(\mu_{x}\right)=1$ for all $x \in U^{*}$ (because $\mu_{x}=\mu_{x}^{h}=\mu_{x h}$ would imply $x=x h$ ). Hence $H=\left\langle\mu_{x} \mu_{y}\right\rangle \leq C_{H}(F(H)) \leq F(H)$, so $H$ is cyclic.

Step 4. $\mathbb{M}(U, \tau) \cong \mathbb{M}(q)$, where $q=|U|$.

Sketch of proof of Step 4. We pick $e \in U^{*}$ and we let $\tau=\mu_{e}$. We show that $H=\left\{h_{a} \mid a \in U^{*}\right\}$, so $|H|=q-1$. We then define

$$
a \cdot b=a h_{b}^{q / 2}
$$

and we show that $(U,+, \cdot, e)$ is a field and that $\tau$ is the inverse map of this field.

This completes the proof of Theorem 7.7.2.

In the case where $|U|$ is odd a theorem similar to Theorem 7.7.2 holds, we state it but omit the (more involved) discussion on it.

Theorem 7.7.3. Let $\mathbb{M}(U, \tau)$ be a finite special Moufang set such that $|U|=q$ is odd. Then $q$ is a power of a prime $p, U$ is elementary abelian and $\mathbb{M}(U, \tau) \cong \mathbb{M}(q)$.

Proof. See [S]. 


\section{References}

[BN] A. Borovik and A. Nesin, Groups of finite Morley rank, Oxford university press, London, 1994.

[BrKl] R. H. Bruck and E. Kleinfeld, The structure of alternative division rings, Proc. Amer. Math. Soc. 2 (1951), 878-890.

[DS] T. De Medts and Y. Segev, Identities in Moufang sets, Trans. Amer. Math. Soc. 360 (2008), 5831-5852.

[DS2] , Finite special Moufang sets of even characteristic, Commun. Contemp. Math. 10 (2008), no. 3, 449-454.

[DST] T. De Medts, Y. Segev and K. Tent, Special Moufang sets, their root groups, and their $\mu$-maps, Proc. London Math. Soc. 96 (2008), no. 3, 767-791.

[DW] T. De Medts and R. M. Weiss, Moufang sets and Jordan division algebras, Math. Ann. 335 (2006), no. 2, 415-433.

[DW2] _ , The norm of a Ree group, Nagoya Math. J., to appear.

[HKSe] C. Hering, W. M. Kantor and G. M. Seitz, Finite groups with a split BN-pair of rank 1, I, J. Algebra 20 (1972), 435-475.

[Kl] E. Kleinfeld, Alternative division rings of characteristic 2, Proc. Natl. Acad. Sci. USA 37 (1951), 818-820.

[Mc1] K. McCrimmon, A general theory of Jordan rings, Proc. Natl. Acad. Sci. USA 56 (1966), 1072-1079.

[Mc2] - A taste of Jordan algebras, Springer-Verlag, Berlin, Heidelberg, New York, 2004.

[McZ] K. McCrimmon and E. Zel'manov, The structure of strongly prime quadratic Jordan algebras, Adv. Math. 69 (1988), no. 2, 133-222.

[M] B. Mühlherr, Some contributions to the theory of buildings based on the gate property, Ph.D. thesis, Tübingen, 1994.

[MV] B. Mühlherr and H. Van Maldeghem, Moufang sets from groups of mixed type, J. Algebra 300 (2006), no. 2, 820-833.

[P] T. Peterfalvi, Sur les BN-paires scindées de rang 1, de degré impair, Comm. Algebra 18 (1990), no. 7, 2281-2292. 
